\title{
ORIGINAL ARTICLE \\ Clinical efficacy of intravesical electrostimulation on incomplete spinal cord patients suffering from chronic neurogenic non-obstructive retention: a 15-year single centre retrospective study
}

\author{
G Lombardi, M Celso, M Mencarini, F Nelli and G Del Popolo
}

Objective: To evaluate the clinical and urodynamic impact of intravesical electrostimulation (IVES) on incomplete spinal cord injury $(\mathrm{SCl})$ patients suffering from chronic neurogenic non-obstructive urinary retention (N-NOR).

Methods: One-hundred and two patients underwent at least 28 consecutive daily IVES sessions because objective evidence of detrusor acontractility instead of hypocontractility was detected. Diary entries written at various stages by each patient were compared (7 days before the IVES cycle, 15-21 days into the cycle and 7 days before its end). Responders were patients with a mean 50\% reduction in both the number of daily catheterizations and post-void residual urine. Responders underwent further urodynamics at the end of the IVES cycle; patients experiencing first sensation of bladder filling, and the mean volume of first sensation of bladder filling per $\mathrm{ml}, \mathrm{Qmax} \mathrm{mls}^{-1}$, among others, were evaluated. Nineteen individuals who repeated another IVES round were included in this study.

Results: Thirty-eight subjects (37.2\%) responded to IVES and of those, $83.3 \%$ recovered the first sensation of bladder filling after the IVES round. Nineteen responders repeated IVES within 1 year, owing to loss of efficacy. They obtained similar voiding symptoms improvement and urodynamic results as after the first IVES cycle. A timespan of $<2$ years from SCI to IVES, and the presence of first sensation of bladder filling at baseline represented significant predictive parameters for IVES success $(P<0.05)$ using $\chi^{2}$-test.

Conclusions: IVES represents a possible therapeutic option for incomplete $\mathrm{SCl}$ patients with N-NOR.

Spinal Cord (2013) 51, 232-237; doi:10.1038/sc.2012.120; published online 13 November 2012

Keywords: intravesical electrostimulation; neurogenic; aseptic intermittent catheterizations; neurogenic non-obstructive retention

\section{INTRODUCTION}

Neurogenic non-obstructive urinary retention (N-NOR) due to spinal cord injury (SCI) may generate therapeutic problems for the urologist because medical therapies are generally ineffective and patients may also experience recurrent urinary tract infections with possible upper urinary tract impairment. ${ }^{1}$ Therefore, the majority of these patients are forced to use aseptic intermittent catheterizations, suprapubic catheters or long-term indwelling catheterization.

There are several treatment options for N-NOR such as pelvic floor re-education, or drugs like alpha-blockers and parasympathomimetics, although the literature has reported serious possible side effects for the latter., ${ }^{2,3}$ Another conservative approach is intravesical electrical stimulation (IVES) used for patients with neurogenic bladder, including those with incomplete SCI, to increase their bladder sensations and improve bladder emptying. ${ }^{4-7}$

The aim of the study was to report the results of 15 years of IVES administered to patients with incomplete SCI and N-NOR.

\section{MATERIALS AND METHODS}

After receiving approval from our internal ethical committee, a retrospective review of incomplete SCI patients suffering from N-NOR and treated with IVES at our neuro-urology department was performed.
Data were retrieved from our neuro-urology admissions database via a computerized search using the key words: 'neurogenic non-obstructive urinary retention' and 'IVES'. Inpatient data were available over a 15-year period (September 1996 to September 2011). We selected only patients with incomplete SCI according to the American Spinal Injury Association (ASIA) Impairment Scale (AIS). ${ }^{8}$ Subsequently, we were able to access their demographic information, diagnostic investigations and voiding diaries completed at various stages: pre-IVES and during IVES treatment.

Results are also provided for patients who responded to the first IVES cycle, lost efficacy and then underwent a further IVES cycle. In these cases, voiding diaries provided from follow-ups post first IVES cycle were also available. Scheduled office visits following the first IVES treatments took place at 3 months, 6 months and then every 6 months, as well as by request if patients experienced worsening or loss of voiding symptoms.

During an office visit, patients had their complete history taken and underwent a physical examination, urine culture, creatinine serum levels, urethrocystoscopy and video-urodynamic study. Only patients with objective evidence of N-NOR were included (Table 1).

Seven days before starting the IVES cycle each patient completed a 1-week voiding diary reporting the number of daily catheterizations, post-void residual (PVR) urine and voiding volume $\mathrm{ml}^{-1}$ per void. During the IVES procedure each diary entry was compared with baseline at 15-21 days and 7 days before to the end of the IVES round. Responders were those with a minimum of $50 \%$ reduction in both the number of daily catheterizations and PVR urine. 
Table 1 Reports of the inclusion criteria

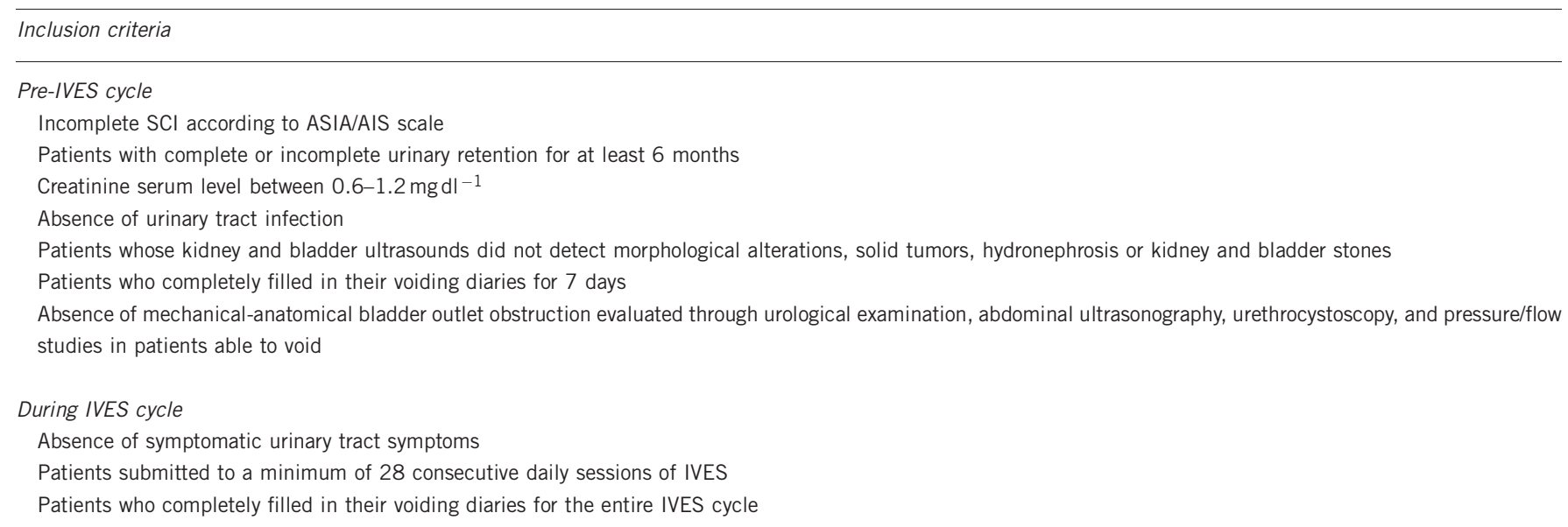

Abbreviations: ASIA/AIS, American Spinal Injury Association (ASIA) Impairment Scale (AIS); IVES, intravesical electrostimulation.

Following the last IVES session, urodynamics were administered again only to responders. Urodynamic patterns, compared with baseline, were: presence of first sensation of bladder filling, volume of first sensation of bladder filling per ml, P det Qmax cm per $\mathrm{H}_{2} \mathrm{O}$, Qmax $\mathrm{mls}^{-1}$, maximum bladder capacity per ml, detrusor compliance $\mathrm{ml}$ per $\mathrm{cm}_{2} \mathrm{O}$ and PVR urine volume.

\section{Description of video-urodynamics}

Video-urodynamic study included continuous filling cystometry at a rate of $20 \mathrm{ml} \mathrm{min}{ }^{-1}$ with maximum filling of $500 \mathrm{ml}$. During cystometry, all SCI patients were asked to report any and all sensations related to bladder filling: initial sensation, first desire to void and strong desire to void.

Pressure/flow studies were reported for patients able to void with concomitant assessment of detrusor sphincter dyssynergia through a surface electromyography located at the perineum. ${ }^{9}$ A 6 -fr catheter with separated double lumen was used.

Bladder contractility index $(\mathrm{BCI})$ was calculated $=(\mathrm{PdetQmax}+5 \mathrm{Qmax})$. According to the $\mathrm{BCI}$, detrusor contractility was categorized as weak $(<100)$, normal $(100-150)$ and strong $(>150) .{ }^{10}$ PVR urine volume was evaluated as well.

\section{Description of the IVES procedure}

The IVES procedure was performed using either the Uroplus A 20 device or the Urotrain $\mu \mathrm{P}$ controlled type: bio-feedback both from SI.EM (Società Italiana Elettro-Medicali) Milan, Italy. These devices were programmed with the same stimulation parameters. A monopolar active electrode was inserted into a catheter $(10-12 \mathrm{Ch})$ and placed in the bladder, while one neutral electrode was applied to skin with preserved sensitivity. Sterile saline solution instillation measuring a third of the patient's bladder capacity was used. Continuous cystomanometry was performed during each IVES session. Each session took 90 minutes.

\section{IVES parameters setting}

Parameters settings specific to each patient were researched during cystomanometry in order to increase or recover bladder sensation, favor detrusor contraction and reduce PVR urine, thus allowing operators to vary intensity, frequency, duration of pulse, interval between pulse trans and finally, increase to maximum intensity of stimulation. Intermittent stimulation and monophasic rectangular impulse were consistently used throughout the IVES cycles.

The intensity varied from $2-25 \mathrm{~mA}$, frequency from 10 to $70 \mathrm{~Hz}$, duration of pulse between $2-5 \mathrm{~ms}$, interval between pulse trans from 3 to $6 \mathrm{~s}$, and finally, increase to maximum intensity of stimulation in the range from 2 to $6 \mathrm{~s}$.
Table 2 Reports of the main characteristics of the patients included in the study

\begin{tabular}{lcc} 
Parameters & $\begin{array}{c}\text { Non-responders 64 } \\
\text { patients (\%) }\end{array}$ & $\begin{array}{c}\text { Responders 38 } \\
\text { patients (\%) }\end{array}$ \\
\hline $\begin{array}{l}\text { Number of males } \\
\text { Age at the time of IVES treatment }\end{array}$ & $\begin{array}{l}49(76.5) \\
\text { > } 40 \text { years }\end{array}$ & $28(73.6)$ \\
Level of SCl: Thoracic 11-Lumbar 5 & $64(100)$ & $38(100)$ \\
& & \\
(ASIA)/(AIS) & $7(10.94)$ & $3(7.89)$ \\
B & $34(53.12)$ & $19(50)$ \\
C & $23(46.94)$ & $16(42.11)$ \\
D & & \\
Etiology of SCI & $43(67.19)$ & $25(65.79)$ \\
Traumatic & $11(17.19)$ & $6(15.79)$ \\
Myelitis & $6(9.4)$ & $5(13.16)$ \\
Vascular & $4(6.2)$ & $2(5.26)$ \\
Others & $42(65.62)$ & $19(50)$ \\
Patients with complete urinary retention & $64(100)$ & $38(100)$ \\
Use of oral alfaltic drugs pre-IVES &
\end{tabular}

Abbreviations: ASIA/AIS, American Spinal Injury Association (ASIA) Impairment Scale (AIS); IVES, intravesical electrostimulation; SCI, spinal cord injury.

\section{Statistical tests performed}

In the statistical analysis of the data, only those values with $P<0.05$ were considered significant.

The $\chi^{2}$-test was used to compare responders and non-responders regarding the following variables at baseline: the number of patients who reported first sensation of bladder filling and the time elapsed from SCI to IVES treatment. The Wilcoxon paired test was used on the group of patients who repeated another IVES cycle, comparing both the mean values of each diary entry from the last week of the 2 IVES cycles, and the mean values of the following urodynamic parameters obtained at the end of each IVES cycle: first sensation of bladder filling, $\mathrm{PQmax} \mathrm{cm} / \mathrm{H}_{2} \mathrm{O}$ and Qmax $\mathrm{ml} / \mathrm{s}$.

\section{RESULTS}

Overall, 115 patients with incomplete SCI who had suffered from N-NOR for at least 6 months underwent IVES. Thirteen patients were excluded; nine because of improper diary compilation and four due 
to IVES side effects (two for epididymo-orchitis and two women for symptomatic urinary tract infection). These patients did not perform the 28 consecutive daily IVES sessions. A total of 102 patients were included in this retrospective study.

Through IVES, 38 of these 102 patients (37.2\%) attained a minimum reduction of $50 \%$ both in the number of daily catheterizations and in PVR urine at 15-21 days and 7 days before the end of the treatment, and then compared with the entries from the pre-IVES week. These entries indicated that patients responded well to IVES stimulation.

To facilitate analysis of the results, we divided the patients into two groups: responders and non-responders.

\section{IVES non-responders}

Mean number of IVES sessions: 33 days (range 28-44 days). Table 2 reports this group's main characteristics.

Of the 64 patients, $42(65.62 \%)$ demonstrated complete urinary retention and required 4-5 catheterizations per day, while 22 (34.38\%) demonstrated incomplete urinary retention in that they were able to void ( $>50 \mathrm{ml}$ per void) with high PVR urine volume of at least $200 \mathrm{ml}$, and perform between 2 and 4 catheterizations per day.

The main urodynamic findings at baseline are shown in Table 3. No detrusor overactivity was detected in any patient. Of the 22 spontaneous voiders, pressure/flow studies showed bladder emptying via the Valsalva maneuver with vesical pressure between 72 and 103, and the BCI was $<100$. No detrusor sphincter dyssinergia was revealed in any patient. No patients modified their initial ASIA/AIS class at the end of the IVES cycle.

\section{IVES responders}

The characteristics of these 38 individuals $(37.2 \%)$ are noted in Table 2.

Table 3 Main urodynamic findings at baseline

\begin{tabular}{|c|c|c|}
\hline Urodynamics & $\begin{array}{c}\text { Non responders } 64 \\
\text { patients (\%) }\end{array}$ & $\begin{array}{c}\text { Responders } 38 \\
\text { patients (\%) }\end{array}$ \\
\hline \multicolumn{3}{|l|}{ Cystometric phase } \\
\hline $\begin{array}{l}\text { Number of patients who referred first } \\
\text { sensation of bladder filling }\end{array}$ & $11(17.19)$ & $14(36.84)$ \\
\hline $\begin{array}{l}\text { Mean volume of first sensation of } \\
\text { bladder filling per } \mathrm{ml}\end{array}$ & 352.72 & 338.90 \\
\hline Compliance $\geqslant 50 \mathrm{ml} \mathrm{cm}^{-1} \mathrm{H}_{2} \mathrm{O}$ & $64(100)$ & $38(100)$ \\
\hline Capacity of filling of $500 \mathrm{ml}$ & $64(100)$ & $38(100)$ \\
\hline \multicolumn{3}{|l|}{ Voiding phase } \\
\hline Number of patients able to void & $22(34.37)$ & $19(50)$ \\
\hline Mean Pdet Qmax cm per $\mathrm{H}_{2} \mathrm{O}$ & 19.95 & 21.10 \\
\hline Mean Qmax mls ${ }^{-1}$ & 4.31 & 4.47 \\
\hline Post-void residual urine per $\mathrm{ml}$ & 310.90 & 304.73 \\
\hline
\end{tabular}

Of the 38 patients, 19 (50\%) demonstrated complete urinary retention, performing 4-5 catheterizations per day, and 19 demonstrated incomplete urinary retention, able to void $>50 \mathrm{ml}$ per void with a minimum high residual urine of $200 \mathrm{ml}$, requiring between 2 and 4 catheterizations per day.

Mean number of IVES sessions: 35 days (range 28-48 days).

The main urodynamic findings at baseline are shown in Table 3. Detrusor overactivity was not detected in any patient.

Regarding the 19 spontaneous voiders, pressure/flow studies showed bladder emptying through the Valsalva maneuver with vesical pressure between 72 and $91 \mathrm{~cm} \mathrm{H}_{2} \mathrm{O}$, and the BCI was $<100$. All patients had a maximum pdetQmax around $35 \mathrm{~cm} \mathrm{H}_{2} \mathrm{O}$. No detrusor sphincter dyssynergia was documented in any patient.

As for clinical parameters throughout the IVES cycle, compared with baseline, each of the 38 patients reached a concomitant mean reduction of at least $50 \%$ in PVR urine and in the number of catheterizations. (Table 4). Moreover, Table 4 reports the mean voiding volume per $\mathrm{ml}$ value at baseline and at the two follow-ups post IVES cycle.

\section{Post IVES urodynamic findings}

During filling cystometry no detrusor overactivity was detected in any patients. All 38 patients noted first sensation of bladder filling, compared with 14 patients at baseline. The 38 subjects had compliance of $>20$ per $\mathrm{cm} \mathrm{H}_{2} \mathrm{O}$. Only six patients had a maximum bladder capacity of $<500 \mathrm{ml}$ (range $400-450 \mathrm{ml}$ ). Mean PVR residual urine was 118.41 (range 70-200).

The BCI was $>100$ for only $31.6 \%$ of the patients. The others used assisted bladder emptying through the Valsalva maneuver (between 63 and $82 \mathrm{~cm} \mathrm{H}_{2} \mathrm{O}$ ). No patients modified their initial ASIA/AIS class at the end of the IVES cycle.

\section{IVES parameters setting}

Only low frequencies $(10-20 \mathrm{~Hz})$ were used on the responders, while it varied from $10-70 \mathrm{~Hz}$ for the non-responders.

\section{Predictive parameters for IVES success}

Taking into account the time since SCI occurred to receiving IVES treatment, a shorter time from SCI to IVES $(<2$ years versus $>2$ years) represented a significant statistical parameter for the success of IVES $(P<0.05)$ using $\chi^{2}$-test. Of the responder group, 14 versus 11 of the non-responders had success within 2 years. Similarly, the first sensation of bladder filling was reported by 14 of the responders versus 11 non-responders providing a significant statistical parameter for the success of IVES $(P<0.05)$ using $\chi^{2}$-test.

\section{Patients who repeated the IVES procedure}

Nineteen out of 38 responders (50\%) repeated another round of IVES (minimum of 28 daily sessions) within 10 months of the first cycle (range 8-15 months) due to loss of efficacy (with a return to similar baseline voiding symptoms). Only at 3 months did each patient

Table 4 Responders: clinical findings during the two IVES follow-ups

\begin{tabular}{lcc}
\hline Bladder entries & Baseline 7 days pre-IVES & First IVES follow-up (range \% I) \\
\hline Mean post-void residual urine and s.d. & $346.57 \pm 75.31$ & $106 \pm 31(53-80)$ \\
Mean number of AIC per day and s.d. & $3.36 \pm 0.91$ & $0.68 \pm 0.53(50-100)$ \\
Mean voided volume per $\mathrm{ml}$ and s.d. & $47.9 \pm 47.3$ & $185 \pm 19$
\end{tabular}

Abbreviations: AIC, aseptic intermittent catheterisations; I, improvement; IVES, intravesical electrostimulation. 
maintain analogous improvement in their voiding symptoms, compared with the end of IVES, whereas at 6 months eight patients had voiding improvement of $<50 \%$ compared with baseline, and at baseline 2 (range $8-15$ months), similar baseline voiding symptoms had returned to each of the 19 patients (Figure 1). All of these patients maintained the same ASIA/AIS class at the end of the second IVES round as they had before the first cycle.

The mean values of each bladder entry recorded during the last week of the two IVES cycles statistically matched the urodynamic patterns (Figure 2).

Urodynamic findings showed that at the end of the two IVES trials the same six patients had a maximum bladder capacity of $<500 \mathrm{ml}$ (range 400-450 ml). An additional patient had a BCI of $>100$. Mean PVR residual urine was alike, with a value of $95 \mathrm{ml}$ (range 30-130) at the end of the first IVES cycle versus 101 (range 50-150) recorded during urodynamics at the end of the second IVES round.

\section{IVES parameters settings}

Intermittent stimulation and monophasic rectangular impulse were consistently maintained during the IVES procedure. The frequency was set between 10 and $20 \mathrm{~Hz}$.

\section{DISCUSSION}

IVES therapy has not been widely accepted as standard treatment for patients suffering from N-NOR because results in the literature are still equivocal. Several groups evaluating hypocontractile or acontractile bladders have reported regained detrusor activity and increased awareness of bladder filling after IVES. ${ }^{11-14}$ Other authors reported negative findings. ${ }^{15,16}$

The discrepancy between authors on the success rate of IVES may reflect differences both in patient selection and in procedure (IVES treatment protocol). ${ }^{17}$ At this time, inclusion/exclusion criteria for IVES are not defined for neurogenic patients with N-NOR (including patients with SCI). ${ }^{18}$ A predictive parameter emerged from this retrospective study indicating that a shorter elapsed time from injury to IVES treatment ( $<2$ years) increased probability of success. Our SCI population was composed exclusively of individuals with N-NOR who began treatment at least 6 months post injury, because during this interval they may have been able to recover detrusor function spontaneously as reported by Madersbacher et al. ${ }^{11}$ where the improvement on voiding function was not attributable to IVES therapy, but rather to the favorable evolution of neurological SCI. None of the 38 responders in our sample showed neurological improvement throughout the IVES cycle; neither did the 19 responders who repeated another IVES round.

Another predictive parameter for increasing the success rate of IVES comes from the urodynamics done at baseline. In the responding group, a higher percentage of patients experiencing first sensation of bladder filling was noted. Urodynamics performed in this group at the end of the IVES cycle showed that all patients had bladder sensation. This hints that the principle mechanism of IVES is to induce/improve bladder sensation. In fact, an evident improvement
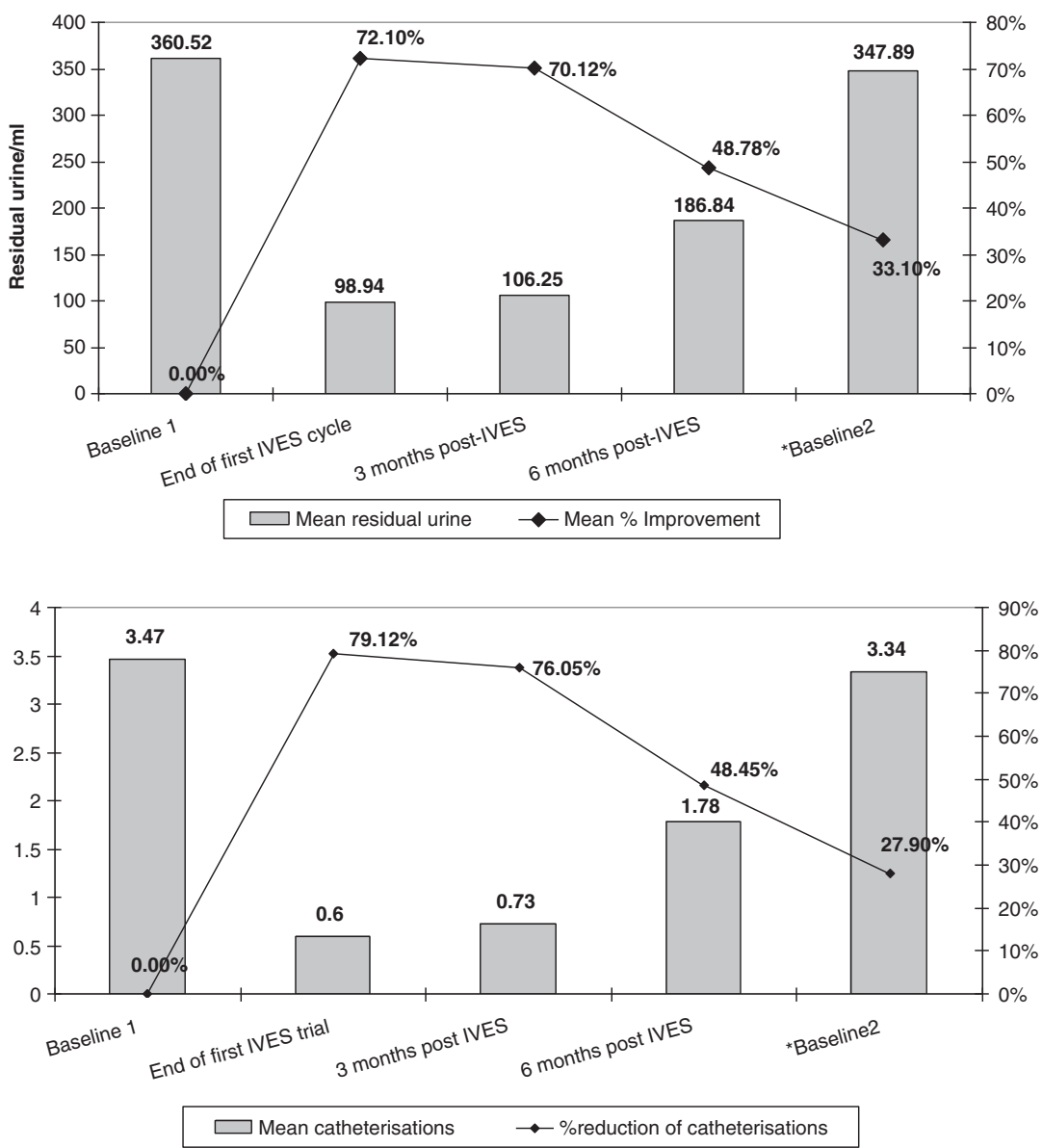

Figure 1 Reports the trends of urinary voiding from baseline 1 up to baseline 2. *Baseline 2: range 8-15 months post the end of first IVES trial. A full color version of this figure is available at the Spinal Cord journal online. 

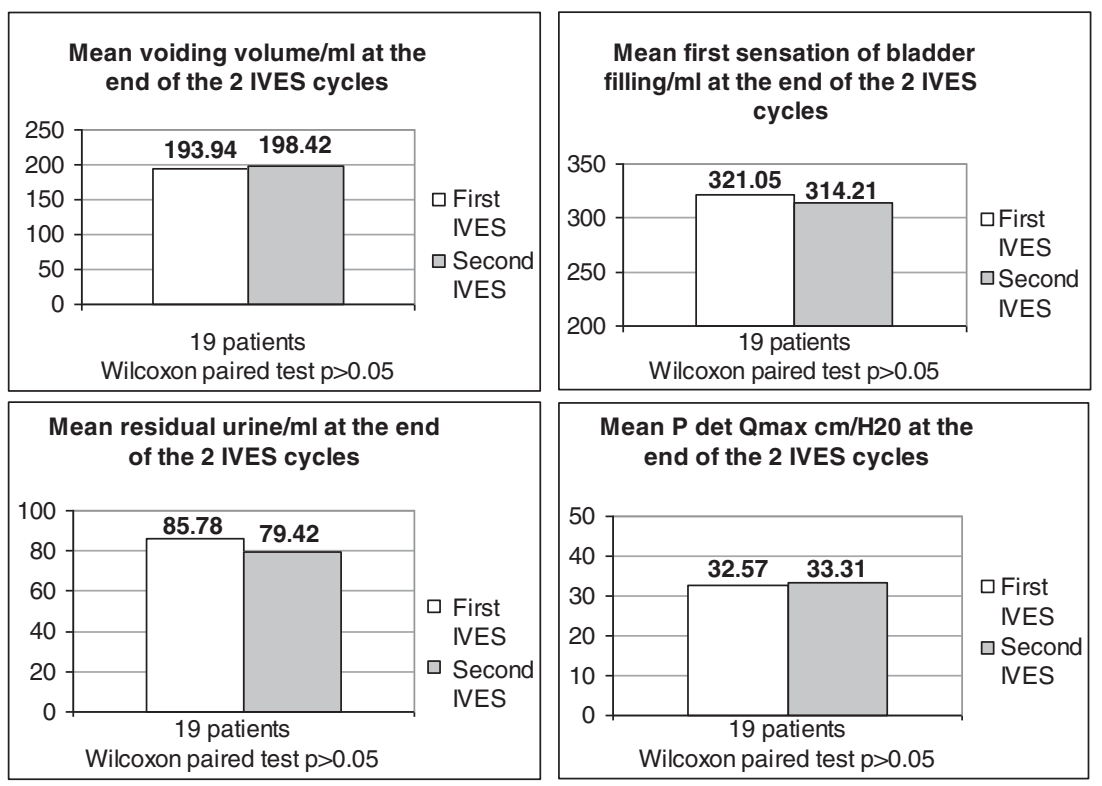
Mean catheterisations at the end
of the 2 IVES cycles

Mean Qmax $\mathrm{ml} / \mathrm{sec}$ at the end of

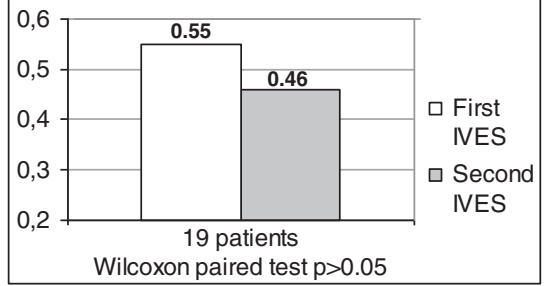
the 2 IVES cycles

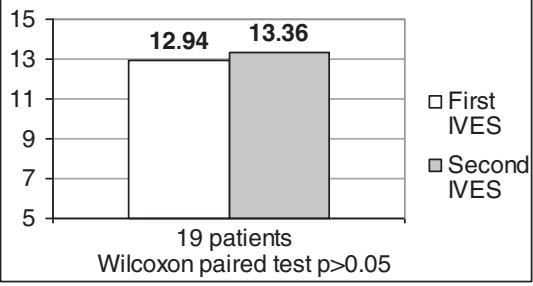

Figure 2 Reports voiding entries and urodynamic patterns at the end of the two IVES cycles.

on bladder contractility was detected in only about $30 \%$ of the responding group, while the other $70 \%$ reported voiding improvement linked to more efficient straining possibly due to the effect of IVES on pelvic floor coordination. Unfortunately, one limitation of our study was neglecting to perform urodynamics on the nonresponders at the end of IVES, thereby making it impossible to compare the results of the two groups.

As regards the IVES procedure, our study revealed that 3 weeks of daily IVES sessions are enough to either manifest notable voiding improvements or confirm failure. In fact, all responders showed no further notable voiding improvement after 3 weeks of IVES stimulation, just as no patient started to respond after 3 weeks of IVES sessions. What is more, it was observed that low frequency was used for all responders, as shown in other reported N-NOR studies. ${ }^{14,19}$

IVES is safe; in fact, only a low percentage $(3.5 \%)$ of mild side effects was detected, although it's virtually impossible to assess minor complications retrospectively by chart reviews. ${ }^{11-14}$ Although loss of efficacy was demonstrated in the short term following the first IVES cycle, all 19 patients repeating a second round attained the same notable improvement in voiding symptoms as the first.

Considering our findings, IVES represents a possible therapeutic option for incomplete SCI patients with N-NOR, taking into account that IVES treatment represents, even at this time, the only available conservative option to induce/improve bladder sensation, and to enhance micturition reflex in patients with incomplete central or peripheral nerve damage. ${ }^{6,7}$

Finally, considering that patients are otherwise forced into life-long aseptic intermittent catheterizations, ongoing investigation into basic research on the theoretical understanding of the working mechanism of IVES might help to improve patient selection, optimize stimulation parameters (with possible shorter IVES sessions), and extend the treatment's duration efficacy, leading to a cost-effective treatment. ${ }^{20}$

\section{DATA ARCHIVING}

There were no data to deposit.

\section{CONFLICT OF INTEREST}

The authors declare no conflict of interest.

1 White WH, Doggweiler R, Klein FA. Surgical management of non-obstructive urinary retention. Minerva Urol Nefrol 2008; 60: 85-92.

2 Linsenmeyer TA, Horton J, Benevento J. Impact of alpha1- blockers in men with spinal cord injury and upper tract stasis. J Spinal Cord Med 2002; 25: 124-128.

3 Barendrecht MM, Oelke M, Laguna MP, Michel MC. Is the use of parasympathomimetics for treating an underactive urinary bladder evidence-based? BJU Int 2007; 99 . 749-752

4 Kaplan WE, Richards I. Intravesical transurethral electrotherapy for neurogenic bladder. J Urol 1986; 136: 243-246.

5 Wyndaele JJ, Madersbacher $\mathrm{H}$, Kovindha A. Conservative treatment of the neuropathic bladder in spinal cord injured patients. Spinal Cord 2001; 39: 294-300.

6 Madersbacher $\mathrm{H}$. Intravesical electrical stimulation for the rehabilitation of the neuropathic bladder. Paraplegia 1990; 28: 349-352.

7 Wyndaele JJ. Conservative treatment of patients with neurogenic bladder. Euro Urol Supplements 2008; 7: 557-565.

8 American Spinal Injury Association. International Standards for Neurological and Functional Classification of Spinal Cord Injury Revised 1996. American Spinal Injury Association: Chicago, 1996. 
9 Schurch B, Schmid DM, Karsenty G, Reitz A. Can neurologic examination predict type of detrusor sphincter-dyssynergia in patients with spinal cord injury? Urology 2005; 65: 243-246.

10 Abrams P. Bladder outlet obstruction index, bladder contractility index and bladder voiding efficiency: three simple indices to define bladder voiding function. BJU Int 1999; 84: 14-15.

11 Madersbacher $\mathrm{H}$, Hetzel H, Gottinger F, Ebner A. Rehabilitation of micturition in adults with incomplete spinal cord lesions by intravesical electrotherapy. Neurourol Urodyn 1987; 6: 230-232.

12 Madersbacher H, Pauer W, Reiner E, Hetzel H, Spanudakis S. Rehabilitation of micturition in patients with incomplete spinal cord lesions by transurethral electrostimulation. Eur Urol 1982; 8: 111-116.

13 Katona F. Basic principles and results in intravesical electric stimulation. Urodinamica 1992; 1: 57-64.

14 Primus G, Kramer G, Pummer K. Restoration of micturition in patients with acontractile and hypocontractile detrusor by transurethral electrical bladder stimulation. Neurourol Urodyn 1996; 15: 489-497.
15 Boone TB, Roehrborn CG, Hurt G. Transurethral intravesical electrotherapy for neurogenic bladder dysfunction in children with myelodysplasia: a prospective, randomized clinical trial. J Urol 1992; 148: 550-554.

16 Decter RM, Snyder P, Laudermilch C. Transurethral electrical bladder stimulation: a follow-up report. J Urol 1994; 152: 812-814.

17 Jiang $\mathrm{CH}$, Lindstrom S. Optimal conditions for the long-term modulation of themicturition reflex by intravesical electrical stimulation: an experimental study in the rat. BJU Int 1999; 83: 483-487.

18 Stöhrer M, Castro-Diaz D, Chartier-Kastler E, Kramer G, Mattiasson A, Wyndaele JJ. Guidelines on neurogenic lower urinary tract dysfunction. Eur Urol. February 2003: $1-40$.

19 De Wachter S, Wyndaele JJ. Quest for standardisation of electrical sensory testing in the lower urinary tract: the influence of technique related factors on bladder electrical thresholds. Neurourol Urodyn 2003; 22: 118-122.

20 De Bock F, De Wachter S, Wyndaele JJ. Influence of nerve transsections and combined bladder filling on intravesical electrostimulation-induced bladder contraction in the rat. Eur Urol 2009; 56: 527-533. 